\title{
To print or not to print, that is the question: how close are we to clinical translation of contemporary bioinks?
}

\author{
Stuart Kyle*,1,2 \& lain S Whitaker ${ }^{* *}, 1,2$ \\ ${ }_{1}^{1}$ Reconstructive Surgery \& Regenerative Medicine Research Group (www.ReconRegen.com), Institute of Life Sciences, Swansea \\ University Medical School, Swansea, SA2 8PP, UK \\ ${ }^{2}$ The Welsh Centre for Burns \& Plastic Surgery, Morriston Hospital, Swansea, SA6 6NL, UK \\ * Author for correspondence: stuart.kyle@doctors.org.uk \\ ** Author for correspondence: iainwhitaker@fastmail.fm
}

"Continual advances in medical knowledge, engineering and technology means 3D bioprinting has the potential to revolutionize personalized medicine within a generation. The fabrication of complex, heterogeneous and multicellular constructs, at scales with clinical relevance, is an exciting prospect for the future."’

First draft submitted: 28 September 2017; Accepted for publication: 17 November 2017; Published online: 15 December 2017

Keywords: 3D printing • biofabrication • bioprinting • printability • print resolution • shape fidelity • structural integrity

Despite the continual addition of names to organ transplant registers worldwide, and the many millions of individuals with tissue loss resulting in structural, cosmetic and functional problems, there is still no sustainable solution. One promising approach to alleviate these problems and revolutionize current medical treatments is biofabrication, which over the last decade has rapidly evolved from a niche research area into more mainstream, integrated bioprinting platforms. The success of this shift has been attributed to advances in engineering of bioprinters using automated robotics that have enabled bioinks to be temporo-spatially deposited layer-by-layer in $3 \mathrm{D}$ in a precise manner. Certain systems have become more economically viable for custom-made production and, in combination with advances in $3 \mathrm{D}$ medical imaging, offer the potential to replace tissues and organs in a scalable, patient-specific approach revolutionizing personalized medicine.

Biofabrication falls within the multidisciplinary field of tissue engineering that aims to create or restore tissues and/or organs through hierarchical assembly of cells, bioactive/biofunctional molecules and biomaterials/bioinks into well-defined composite structures. This approach relies on careful interplay between the 'printability' of biomaterials, the ability to incorporate cells and enhance viability and durability. Next-generation bioinks are now being designed to improve shape and print fidelity through careful control of printing parameters while maintaining physicochemical and biological properties. We have recently reviewed the current state of play in understanding the importance of 'printability' of bioinks in order to dispense small units of cells and biomaterials with micrometer precision and high resolution so that tissue-like structures can be fabricated [1].

In the biofabrication and bioprinting community, we consider 'printability' as the relationship between bioinks and substrates that results in the layer-by-layer additive manufacturing of accurate, well-defined structures with structural fidelity and integrity. The added complexity that comes with the introduction of living cells is the next challenge, as is the delivery in a well-controlled micro- and macro-environment.

The key areas to consider while assessing 'printability' are the manipulation and control of nozzle and printing parameters, and the interaction between the bioink and substrate when forming the first layer. This means that both the printing on the substrate surface and the subsequent 'layer-on-layer' must have tunable properties, and ensure that each layer can withstand forces imposed upon them to maintain structural integrity. A deep insight into the physics of bioprinting is important, to allow understanding of varying contact angles and surface tensions between bioinks and substrates. Coating with thin polymer layers is often used to enhance the printing surface.

Future $\because$ Medicine 
Viscosity and rheology of bioinks are key parameters for successful extrusion-based 3D bioprinting that are gaining further attention in research communities worldwide. As viscosity is determined by bioink concentration, molecular weight and temperature, low viscosity bioinks lead to structural collapse of printed structures. Sufficient viscosity is required to overcome surface tension forces and the formation of continuous extruded filaments. The optimal situation is the formation of separate elegant filaments resulting in cylindrical rather than spheroidal shapes. The refinement and accurate control of shear thinning behavior of bioinks will also be essential in improving print resolution and shape fidelity of complex, $3 \mathrm{D}$ structures. The balance of shear stress and viscosity is the key issue as they are not working synergistically: decreased viscosity and hence shear stress favors cell survival whereas higher viscosities result in print fidelity. The aim is to encapsulate cells with $>95 \%$ viability within bioinks of sufficiently high viscosity deposited via a precise nozzle. Carefully selected bioinks at optimal concentrations and molecular weights lead to better printability while providing enhanced microenvironments for cell differentiation, migration, proliferation and extracellular matrix formation.

A number of strategies have been adopted to address this trade-off between 'printability' and cell biology within complex 3D geometries. Rheological modifiers or 'thickening agents' enhance the viscoelastic properties, which help to improve: filament/line printing; mechanical properties; cell encapsulation and viability; and, pore geometry and structural integrity. Various crosslinking systems have also been employed to allow the extrusion of cell-laden polymer solutions in combination with immediate gelation to improve print fidelity. Contemporary crosslinking techniques involve the use of chemicals, heat, UV light or a mixture of strategies. 'Support baths' and a range of delivery methods, such as spraying, have been used to create an environment conducive to crosslinking. The incorporation of sacrificial materials, such as agarose, gelatin and alginate, is currently being trialed to enhance mechanical support and strength.

Advances in computer simulation, imaging and modelling are incrementally advancing the biofabrication of smart, tunable, responsive and multifunctional materials within the $3 \mathrm{D}$ bioprinting arena. The revolution in $3 \mathrm{D}$ bioprinting by imaging, designing and printing complex geometries is fuelled by increased computational power. Advanced biological imaging is also allowing study of the interaction between the material/cell interface at the 'nano' level.

Due to recent advances in technology, knowledge and equipment, we are currently in a position to image, design and bioprint tissues of clinically relevant and patient-specific sizes. We have the ability to temporo-spatially place multiple cell types in $3 \mathrm{D}$ in order to biomimic the native tissue histoarchitecture. The current major hurdle to clinical translation of 3D printed tissue is durability for their targeted applications. There are many biological and technological strategies being employed to this end, alongside computer simulations to predict changes in structure and function of bioprinted materials.

Although research in the area of $3 \mathrm{D}$ bioprinting is evolving at a rapid pace, there are very few examples of successful clinical translation. The future is exciting, with the prospect of multimaterial bioinks that are smart, adaptive and multifunctional. Through incremental improvements and rationalized design, we expect such materials to maintain excellent 'printability' and shape fidelity alongside enhanced mechanical and biomimetic properties. A better understanding of the surface interactions and functionalization will enhance the physicochemical and biological properties of next-generation bioinks. Other factors impacting clinical translation that must also be considered include degradability, support of vascularization and migration of endogenous progenitor cells, implantability, affordability, commercial availability and anti-immunogenicity of bioinks. Continual advances in medical knowledge, engineering and technology means 3D bioprinting has the potential to revolutionize personalized medicine within a generation. The fabrication of complex, heterogeneous and multicellular constructs, at scales with clinical relevance, is an exciting prospect for the future. It is definitely a question of 'when' will we see the routine use of $3 \mathrm{D}$ bioprinted tissues and organs clinically, not 'if'.

Financial \& competing interest disclosure

The authors have no relevant affiliations or financial involvement with any organization or entity with a financial interest in or financial conflict with the subject matter or materials discussed in the manuscript. This includes employment, consultancies, honoraria, stock ownership or options, expert testimony, grants or patents received or pending, or royalties.

No writing assistance was utilized in the production of this manuscript. 


\section{References}

1 Kyle S, Jessop Z, Al-Sabah A, Whitaker I. 'Printability' of candidate biomaterials for extrusion based 3D printing: state of the art. Adv. Healthcare Mater. 6(16) doi: 10.1002/adhm.201700264 (2017) 
